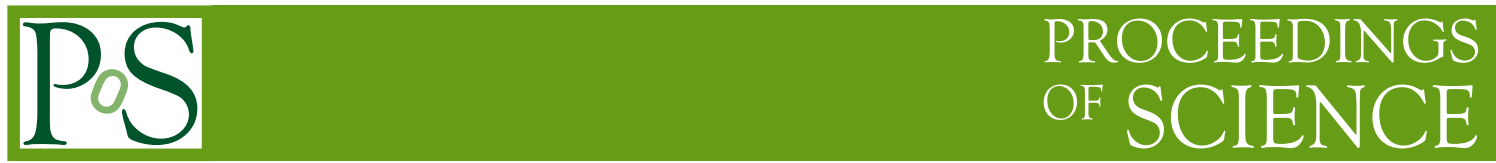

\title{
Review of recent highlights in lattice calculations at finite temperature and finite density
}

\section{P. Petreczky*}

Physics Department, Brookhaven National Laboratory, Upton, NY 11973, USA

E-mail: petreczk@bnl.gov

I review some recent lattice results on studying chiral and deconfinement transition in QCD at finite temperature and density, as well as properties of strongly interacting matter at high temperatures. I discuss lattice results on the equation of state, fluctuations of conserved charges, chromo-electric screening as well as the determination of the chiral transition temperature.

Xth Quark Confinement and the Hadron Spectrum,

October 8-12, 2012

TUM Campus Garching, Munich, Germany

\footnotetext{
* Speaker.

${ }^{\dagger}$ This work was supported by U.S. Department of Energy under Contract No. DE-AC02-98CH10886.
} 


\section{Introduction}

It is expected that strongly interacting matter undergoes a transition in some temperature interval from hadron gas to deconfined state also called the quark gluon plasma (QGP) [1]. Creating and exploring deconfined medium in the laboratory is the goal of the large experimental heavy ion program at RHIC [2] and LHC [3]. Studying hot and dense strongly interacting matter is also the subject of a large effort in lattice QCD (see Refs. [4, 5] for recent reviews). Early lattice QCD simulations at non-zero temperature were limited to large quark masses and had no control over the discretization errors $[6,7,8]$. During the past seven years calculations with the physical strange quark mass and physical or nearly physical light $(u, d)$ quark masses have been performed using improved staggered fermion actions $[9,10,11,12,13,14,15,16,17,18,19]$, and for several quantities continuum extrapolated results have been obtained. There was also progress in lattice QCD calculations at non-zero temperature using other fermion formulations, namely Wilson fermions [20, 21, 22], Domain-Wall fermions [23] and overlap fermions [24]. The later two formulations preserve the chiral symmetry of continuum QCD. However, due to much larger computational costs of these formulations the corresponding results are far less extensive.

To get reliable predictions from lattice QCD the lattice spacing $a$ should be sufficiently small relative to the typical QCD scale, i.e. $\Lambda_{Q C D} a \ll 1$. For staggered fermions, discretization errors go like $\mathscr{O}\left(\left(a \Lambda_{Q C D}\right)^{2}\right)$ but discretization errors due to flavor symmetry breaking turn out to be quite large numerically, and dominate the cutoff dependence of thermodynamic quantities at low temperatures. To reduce these errors one has to use improved staggered fermion actions with so-called fat links [25]. At high temperature the dominant discretization errors come from the lattice distortions of the quark dispersion relation and go like $(a T)^{2}$, and therefore could be very large. Thus, it is important to use improved discretization schemes, which reduce or eliminate these discretization errors. Staggered fermion actions used in numerical calculations typically implement some version of fat links as well as improvement of quark dispersion relation and are referred to as $p 4$, asqtad, HISQ and stout. Independently of specific improvement all lattice results eventually must be extrapolated to the continuum limit.

In this contribution I am going to discuss lattice QCD calculations on the equation of state, study of deconfinement aspects of the QCD transition, including color screening and fluctuations of conserved charges and determination of the chiral transition temperature. I will mostly discuss lattice results obtained with staggered quark formulation; where appropriate results obtained using other actions will be highlighted.

\section{Equation of State}

The equation of state has been calculated using different improved staggered fermion actions p4, asqtad, stout and HISQ. In the lattice calculations of the equation of state and many other quantities the temperature is varied by varying the lattice spacing at fixed value of the temporal extent $N_{\tau}$. The temperature $T$ is given by the lattice spacing and the temporal extent, $T=1 /\left(N_{\tau} a\right)$. Therefore taking the continuum limit corresponds to $N_{\tau} \rightarrow \infty$ at the fixed physical volume. The calculation of thermodynamic observables proceeds through the calculation of the trace of the energy momentum tensor, $\varepsilon-3 p$, also known as the trace anomaly or the interaction measure. 
This is due to the fact that this quantity can be expressed in terms of expectation values of local gluonic and fermionic operators, (see e.g. Ref. [15]). Different thermodynamic observables can be obtained through integration of the trace anomaly ${ }^{1}$. The pressure can be written as

$$
\frac{p(T)}{T^{4}}-\frac{p\left(T_{0}\right)}{T_{0}^{4}}=\int_{T_{0}}^{T} \frac{d T^{\prime}}{T^{\prime 5}}(\varepsilon-3 p) .
$$

The lower integration limit $T_{0}$ is chosen such that the pressure is exponentially small there. Furthermore, the entropy density can be written as $s=(\varepsilon+p) / T$. Since the interaction measure is the basic thermodynamic observable in the lattice calculations it is worth to discuss its properties more in detail. In Fig. 1 (left panel) I show the results of the calculation with different improved actions. The calculation with $p 4$ and asqtad actions have been performed on $N_{\tau}=8$ lattices and light quark masses $m_{l}=m_{s} / 10$, with $m_{s}$ being the physical strange quark mass $[13,15]$. These light quark masses correspond to the pion masses slightly above $200 \mathrm{MeV}$ in the continuum limit. For this value of $N_{\tau}$ the above deviation from the physical quark mass plays little role $[14,26]$. The $N_{\tau}=12$ asqtad calculations have been performed for $m_{l}=m_{s} / 20$ [27]. Calculations with HISQ action have been performed for $N_{\tau}=4,6,8,10$ and 12 for $m_{l}=m_{s} / 20$ corresponding to the pion mass of $160 \mathrm{MeV}$ in the continuum limit [27]. A subset of these results is shown in Fig. 1. Finally, calculation of the trace anomaly and the equation of state was performed with stout action using $N_{\tau}=4,6,8,10$ and 12 and physical light quark masses [18]. Using the lattice data from $N_{\tau}=6,8$ and 10 a continuum estimate for different quantities was given [18]. The interaction measure shows a rapid rise in the transition region and after reaching a peak at temperatures of about $200 \mathrm{MeV}$ decreases. At low temperatures lattice data obtained with HISQ action and stout action agree with each other. We also compare the lattice results with hadron resonance gas (HRG) model calculations which seems to agree well with HISQ and stout results for $T<150 \mathrm{MeV}$. Cutoff effects (i.e. $N_{\tau}$ dependence) appears to be the strongest around the peak region. They decrease at high temperatures and at $T>400 \mathrm{MeV}$ all lattice results agree with each other. At low temperature the cutoff effects related to flavor symmetry breaking are very large for $p 4$ and asqtad actions. Due to these large cutoff effects the $N_{\tau}=8 p 4$ and asqtad data are below the hadron resonance gas model. Taking into account the distortions of the hadron spectrum due to flavor symmetry breaking in hadron resonance gas calculations leads to good agreement of HRG model with the lattice [28]. Since at high temperatures the effects of flavor symmetry breaking in the pressure and the interaction measure are small, the reduction in $\varepsilon-3 p$ at low temperatures must be compensated (at least partly) by a larger value at intermediate temperatures. Thus, the large $N_{\tau}$ dependence of the peak height of $\varepsilon-3 p$ is related to the large flavor symmetry breaking effects for $p 4$ and asqtad actions.

In Fig. 1 I also show the entropy density divided by the corresponding ideal gas value and compare the lattice results with the resummed perturbative calculations $[29,30]$, as well as with the predictions from AdS/CFT correspondence for the strongly coupled regime [31]. The later is considerably below the lattice results. Note that the pressure, the energy density and the trace anomaly have also been recently discussed in the framework of resummed perturbative calculations which seem to agree with lattice data quite well at high temperatures[32]. The differences between the stout action and the $p 4$ and asqtad actions for the trace anomaly translates into the differences

\footnotetext{
${ }^{1}$ A somewhat different approach was used in Ref. [18]
} 

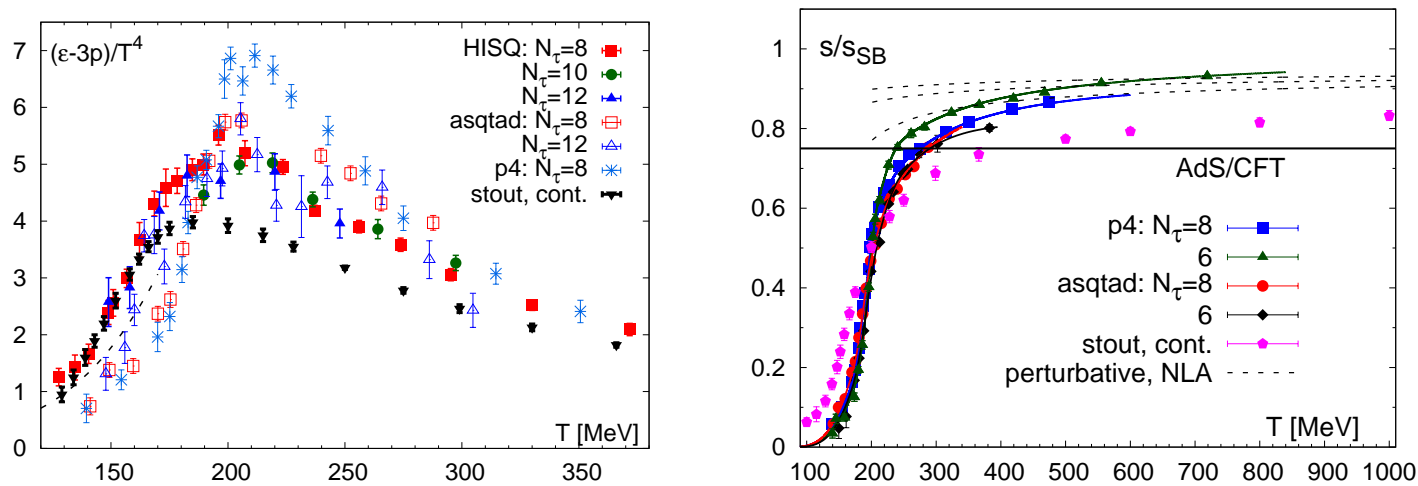

Figure 1: The interaction measure (left) and the entropy density (right) as function of the temperature calculated with improved staggered fermion actions. The dashed line in the left panel shows the HRG result. The band in the right panel shows the resummed perturbative result, while the solid line is the expectations based on the strongly coupled limit.

in the pressure and the entropy density. In the high temperature region the entropy density obtained with stout action is $10 \%$ smaller than the entropy density obtained with $p 4$ and asqtad actions.

\section{Taylor expansion of the pressure and fluctuations of conserved charges}

Due to the infamous sign problem lattice QCD Monte-Carlo simulations are not possible at non-zero quark chemical potentials. The pressure and other quantities at non-zero chemical potentials, however, can be evaluated using Taylor expansion. The Taylor expansion can be set up in terms of the quark chemical potentials $\mu_{u}, \mu_{d}$ and $\mu_{s}$, or in terms of the chemical potentials corresponding to baryon number $B$, electric charge $Q$ and strangeness $S$ of hadrons

$$
\begin{aligned}
\frac{p}{T^{4}} & =\frac{1}{V T^{3}} \ln Z\left(T, \mu_{u}, \mu_{d}, \mu_{s}\right)=\sum_{i j k} \frac{1}{i ! j ! k !} \chi_{i j k}^{u d s}\left(\frac{\mu_{u}}{T}\right)^{i}\left(\frac{\mu_{d}}{T}\right)^{j}\left(\frac{\mu_{s}}{T}\right)^{k} \\
\chi_{i j k}^{u d s} & =\frac{\partial^{i+j+k} p / T^{4}}{\partial\left(\mu_{u} / T\right)^{i} \partial\left(\mu_{d} / T\right)^{j} \partial\left(\mu_{s} / T\right)^{k}} \\
\frac{p}{T^{4}} & =\frac{1}{V T^{3}} \ln Z\left(T, \mu_{B}, \mu_{Q}, \mu_{S}\right)=\sum_{i j k} \frac{1}{i ! j ! k !} \chi_{i j k}^{B Q S}\left(\frac{\mu_{B}}{T}\right)^{i}\left(\frac{\mu_{Q}}{T}\right)^{j}\left(\frac{\mu_{S}}{T}\right)^{k} \\
\chi_{i j k}^{B Q S} & =\frac{\partial^{i+j+k} p / T^{4}}{\partial\left(\mu_{B} / T\right)^{i} \partial\left(\mu_{Q} / T\right)^{j} \partial\left(\mu_{S} / T\right)^{k}} .
\end{aligned}
$$

Using Taylor expansion method the equation of state has been calculated for small chemical potential in the continuum limit [33]. Earlier results at non-zero lattice spacing have been reported in Refs. [34, 35]. While Taylor expansion can be used to study the physics at non-zero baryon density, the expansion coefficients are interesting on their own right as they are related to fluctuations and correlations of conserved charges. As will become clear later fluctuations and correlations of conserved charges are good probes of deconfinement. Fluctuations of conserved charges are also useful for determining the freeze-out conditions in heavy ion experiments [36]. 

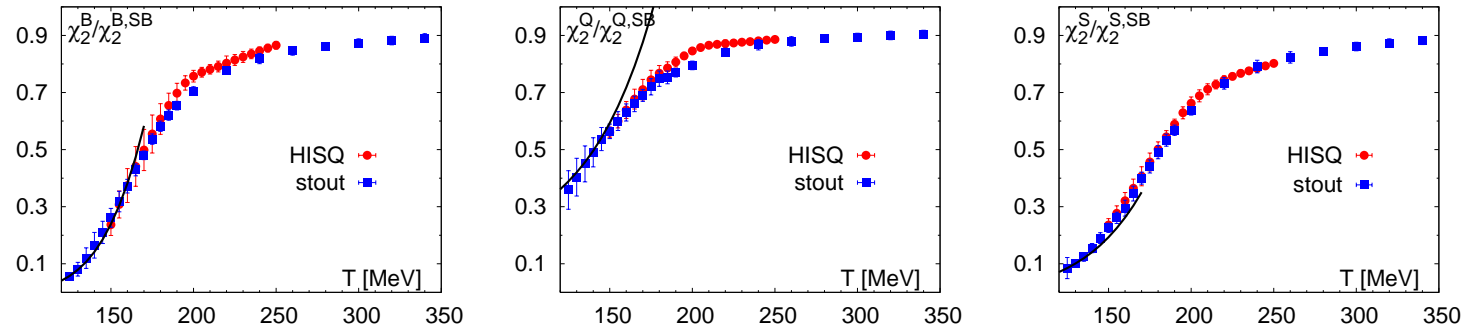

Figure 2: The fluctuations of baryon number (left), electric charge (middle) and strangeness (right) as function of the temperature calculated with $H I S Q$ and stout action in the continuum limit and normalized by the corresponding ideal quark gas values $\chi_{2}^{X, S B}$. The solid black curves correspond to HRG predictions.

The diagonal expansion coefficients are related to second and higher order fluctuations of conserved charges, e.g.

$$
\begin{aligned}
\chi_{2}^{X} & =\frac{1}{V T^{3}}\left\langle N_{X}^{2}\right\rangle \\
\chi_{4}^{X} & =\frac{1}{V T^{3}}\left(\left\langle N_{X}^{4}\right\rangle-3\left\langle N_{X}^{2}\right\rangle^{2}\right),
\end{aligned}
$$

while the off-diagonal expansion coefficients are related to correlations among conserved charges, e.g.

$$
\chi_{11}^{X Y}=\frac{1}{V T^{3}}\left\langle N_{X} N_{Y}\right\rangle .
$$

Second order fluctuations have been studied with improved staggered actions in Refs. [15, 17, 37]. Recently continuum results have been obtained for second order fluctuations of baryon number, electric charge and strangeness using stout and $H I S Q$ actions $[38,39]$ which are shown in Fig. 2. The lattice results are also compared with HRG model. As one can see from the figure at low temperatures the lattice data are described well by HRG model indicating that the dominant degrees of freedom in that temperature range are hadronic. Deconfiniment is seen as a rapid increase of the fluctuations for $T>150 \mathrm{MeV}$, which eventually reach values that are close to the expectations of weakly interacting quark gas. In other words, the fluctuations indicate quark degrees of freedom at high temperatures. The lattice results obtained with HISQ and stout action agree well in the continuum limit, except for temperatures around $200 \mathrm{MeV}$ where some discrepancies are observed in the baryon and electric charge fluctuations. Another way to study deconfinement is to consider correlations of conserved charges. These correlations are very different for hadron gas and quark gas. As an example let us examine the strangeness-baryon number correlation. It is convenient to normalize this quantity as follows $C_{B S}=-3 \chi_{11}^{B S} / \chi_{S}$. At high temperatures where strangeness is carried by s-quarks this quantity should be close to one. At low temperatures on the other hand strange baryons are responsible for strangeness-baryon correlations. In Fig. 3(left) I show continuum results for $C_{B S}$ obtained with stout [38] and HISQ [39] actions. At low temperatures the lattice results are described by HRG model, while at high temperatures they are close to one as expected for quark gas.

Fourth order fluctuations for baryon number, electric charges and strangeness have been studied using $p 4$ [37] and HISQ [40] actions. Cutoff effects are quite large for $p 4$ action in the low 

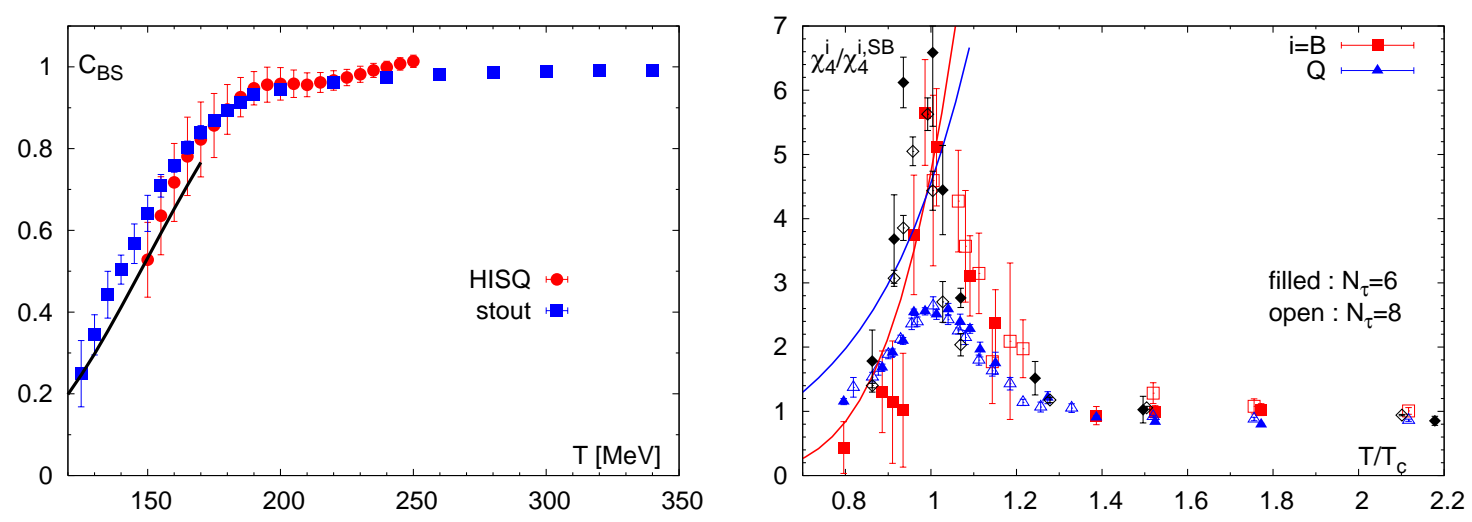

Figure 3: Baryon number-strangeness correlation $C_{B S}$ (left) and fourth order fluctuations of baryon number and electric charge for $H I S Q$ action normalized by the corresponding ideal quark gas value as function of $T / T_{c}$ (right). The lines correspond to the prediction of the hadron resonance gas model. Open (filled) diamonds in the right panel correspond to $N_{\tau}=4 p 4$ results for $\chi_{4}^{Q}\left(\chi_{4}^{B}\right)$.

temperature and the transition regions. These large cutoff effects also result in much larger value of the transition temperature than obtained with stout and HISQ actions [10]. Calculations of the fourth and higher order fluctuations are quite demanding computationally and for this reason no continuum results have been obtained yet. The lattice results obtained with $H I S Q$ action for $N_{\tau}=6$ and 8 are shown in Fig. 3 as function of the temperature in units of the chiral transition temperature $T_{c}=154 \mathrm{MeV}$ (see below) and also compared with earlier results obtained with $p 4$ action and $N_{\tau}=4$. For the $p 4$ action we use the value of the transition temperature $T_{c}=204 \mathrm{MeV}$ determined in Ref. [10] for $N_{\tau}=4$. To reduce the cutoff effects the lattice spacing in the HISQ calculations was set by the kaon decay constant $f_{K}$. At high temperatures the fourth order fluctuations are close to the values corresponding to non-interacting quark gas. At low temperatures the fourth order baryon number fluctuations are reasonably well described by hadron resonance gas. This is not the case, however, for the electric charge. One possible reason for the disagreement between the lattice and HRG model in this case could be the large cutoff effects in the pion sector. Electric charge fluctuations are very sensitive to the pion sector, which is largely distorted on the lattice even if $H I S Q$ or stout action is used. These distortions correspond effectively to a larger pion mass that would explain why the lattice data are below the HRG expectations. Fourth order fluctuations have a maximum in the transition region. Interestingly enough the position of the maximum in $T / T_{c}$ is roughly the same for the HISQ and $p 4$ actions. The height of the peak, however, is significantly larger for the $\chi_{4}^{Q}$ when $p 4$ action is used.

Second and fourth order light and strange quark number fluctuations at high temperatures have been studied on the lattice in Refs. [38, 41] and compared with resummed perturbative calculations $[42,43]$. The resummed perturbative calculations seem to describe the lattice data quite well at $T>300 \mathrm{MeV}$.

\section{Deconfinement : color screening}

One of the most prominent feature of the quark gluon plasma is the presence of chromoelectric 
(Debye) screening. The easiest way to study chromoelectric screening is to calculate the Polyakov loop. The Polyakov loop is an order parameter for the deconfinement transition in pure gauge theory, which is governed by $Z(N)$ symmetry. For QCD this symmetry is explicitly broken by dynamical quarks. There is no obvious reason for the Polyakov loop to be sensitive to the singular behavior close to the chiral limit, although speculations along these lines have been made [45]. The Polyakov loop is related to the screening properties of the medium and thus to deconfinement. After proper renormalization, the square of the Polyakov loop characterizes the long distance behavior of the static quark anti-quark free energy; it gives the excess in free energy needed to screen two well-separated color charges. The renormalized Polyakov loop, calculated on lattices with temporal extent $N_{\tau}$, is obtained from the bare Polyakov

$$
L_{\text {ren }}(T)=z(\beta)^{N_{\tau}} L_{\text {bare }}(\beta)=z(\beta)^{N_{\tau}}\left\langle\frac{1}{3} \operatorname{Tr} W(\vec{x})\right\rangle, W(\vec{x})=\prod_{x_{0}=0}^{N_{\tau}-1} U_{0}\left(x_{0}, \vec{x}\right),
$$

where $U_{0}=\exp \left(i g a A_{0}\right)$ denotes the temporal gauge link and $z(\beta)$ is the renormalization constant determined from the $T=0$ static potential [11]. Continuum results for the renormalized Polyakov loop have been obtained with stout [17] and HISQ actions [48]. These are shown in Fig. 4 together with $N_{\tau}=6$ HISQ results [19]. One can see a good agreement between the stout and HISQ results. I also compare the $2+1$ flavor QCD results with the corresponding results in pure gauge theory [46, 47] as well as with the prediction of non-interacting gas of static-light(strange) hadrons [48, 49]. We see that in the vicinity of the transition temperature the behavior of the renormalized Polyakov loop in QCD and in the pure gauge theory is quite different. The calculation of $L_{r e n}$ based on noninteracting static-light hadron gas can explain the lattice data for $T<140 \mathrm{MeV}$. The renormalized Polyakov loop has also been calculated using lattice fermion formulations other than staggered, namely the Wilson formulation [20] and the overlap formulation [24]. These formulations are considerably more expensive computationally than the staggered formulation and therefore the calculations have been performed at unphysical pion mass. The results obtained using Wilson action and overlap action for the Polyakov loop agree very well with the staggered fermion results at the same value of the pion masses [20,24].

Further insight on chromoelectric screening can be gained by studying the singlet free energy of static quark anti-quark pair (for reviews on this see Ref. [50, 51]), which is expressed in terms of the correlation function of temporal Wilson lines in Coulomb gauge

$$
\exp \left(-F_{1}(r, T) / T\right)=\frac{1}{3} \operatorname{Tr}\left\langle W(r) W^{\dagger}(0)\right\rangle
$$

Instead of using the Coulomb gauge the singlet free energy can be defined in gauge invariant manner by inserting a spatial gauge connection between the two Wilson lines. Using such definition the singlet free energy has been calculated in $S U(2)$ gauge theory [52]. It has been found that the singlet free energy calculated this way is close to the result obtained in Coulomb gauge [52]. The singlet free energy turned out to be useful to study quarkonia binding at high temperatures in potential models (see e.g. Ref. [44] and references therein). It also appears naturally in the perturbative calculations of the Polyakov loop correlators at short distances [53].

The singlet free energy has been recently calculated in 2+1 flavor QCD with $H I S Q$ action on $24^{3} \times 6$ and $16^{3} \times 4$ lattices [54]. The numerical results are shown in Fig. 4. At short distances the 

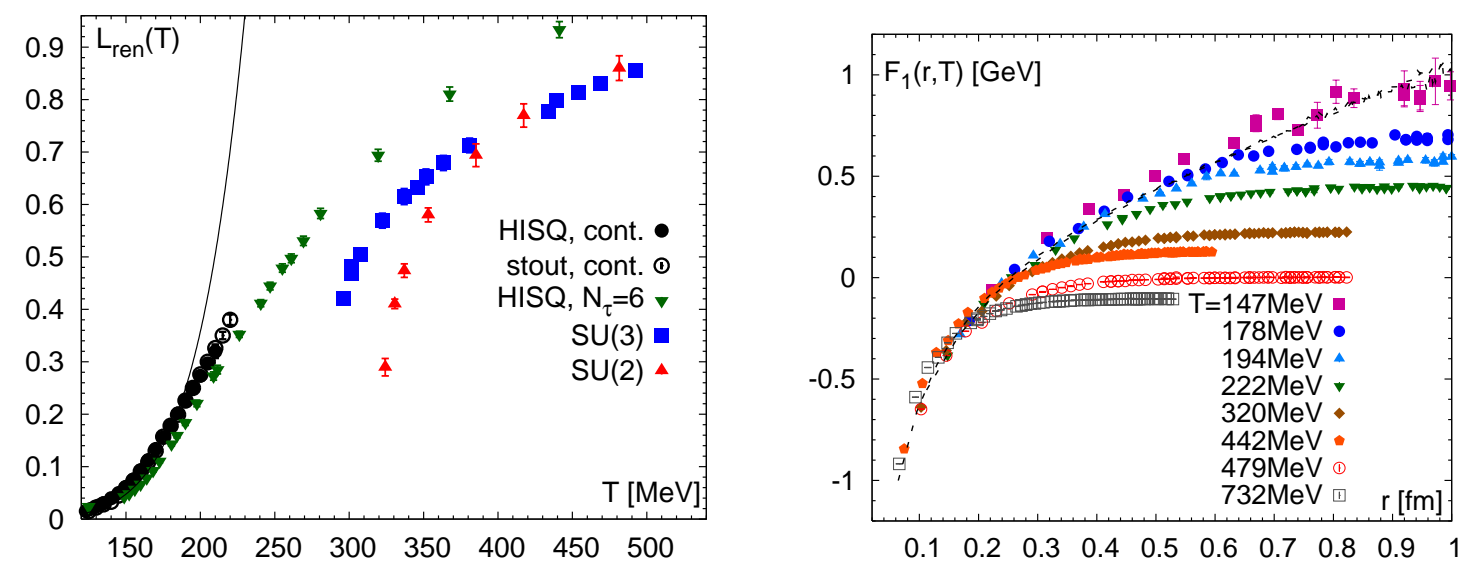

Figure 4: Left: The renormalized Polyakov loop as function of the temperature in $2+1$ flavor QCD and pure gauge theory. Right: The singlet free energy as function of the distance at different temperature calculated with HISQ action [54]. The solid line in the left panel corresponds to static-ligh(strange) hadron gas prediction for $L_{r e n}$ (see text). The dashed line in the right panel is the $T=0$ potential [19].

singlet free energy is temperature independent and coincides with the zero temperature potential. In purely gluonic theory the free energy grows linearly with the separation between the static quark and anti-quark in the confined phase. In presence of dynamical quarks the free energy is saturated at some finite value at distances of about $1 \mathrm{fm}$ due to string breaking (see e.g. Ref. [50]). This is also seen in Fig. 4. Above the deconfinement temperature the singlet free energy is exponentially screened at sufficiently large distances $[46,47]$ with the screening mass proportional to the temperature, i.e.

$$
F_{1}(r, T)=F_{\infty}(T)-\frac{4}{3} \frac{g^{2}(T)}{4 \pi r} \exp \left(-m_{D}(T) r\right), m_{D} \sim T .
$$

The lattice data for the singlet free energy are consistent wit these expectations for $r>0.8 / T$.

Let me finally note that contrary to the electro magnetic plasma the static chormomagnetic fields are screened in QGP. This is due to the fact that unlike photons gluons interact with each other (the stress tensor is non-linear in QCD). Magnetic screening is non-perturbative, i.e. it does not appear at any finite order of pertubation theory. In lattice calculations chromomagnetic screening is studied either in terms of spatial Wilson loops [55] or in terms of spatial gluon propagators $[56,57,58]$. The numerical results obtained so far show that the length scale related to magnetic screening is larger than the one related to electric screening.

\section{Chrial transition}

The Lagrangian of QCD has an approximate $S U_{A}(3)$ chiral symmetry. This symmetry is broken in the vacuum. The chiral symmetry breaking is signaled by non-zero expectation value of the quark or chiral condensate, $\langle\bar{\psi} \psi\rangle \neq 0$ in the massless limit. This symmetry is expected to be restored at high temperatures and the quark condensate vanishes. There is an explicit breaking of the chiral symmetry by the non-zero values of $u, d$ and $s$ quark masses. While due to the relatively large strange quark mass $\left(m_{s} \simeq 100 \mathrm{MeV}\right) S U_{A}(3)$ may not be a very good symmetry its subgroup 
$S U_{A}(2)$ remains a very good symmetry and is relevant for the discussion of the finite temperature transition in QCD. If the relevant symmetry is $S U_{A}(2)$ the chiral transition is expected to be second order for massless light ( $u$ and $d$ ) quarks belonging to the $O(4)$ universality class. Recent calculations with $p 4$ action support this pictures [59]. This also means that for non-zero light quark masses the transition must be a crossover. The crossover nature of the transition is supported by calculations in Ref. [60]. The $U_{A}(1)$ symmetry is explicitly broken in the vacuum by the anomaly but it is expected to be effectively restored at high temperatures as non-perturbative vacuum fluctuations responsible for its breaking are suppressed at high temperatures. If the $U_{A}(1)$ symmetry is restored at the same temperature as the $S U_{A}(2)$ symmetry the transition could be first order [61]. Recent calculations with staggered [62] as well as with domain wall fermions [23] suggest that $U_{A}(1)$ symmetry gets effectively restored at temperature that is significantly higher than the chiral transition temperature.

For massless quark the chiral condensate vanishes at the critical temperature $T_{c}^{0}$ and is the order parameter. Therefore in the lattice studies one calculates the chiral condensate and its derivative with respect to the quark mass called the chiral susceptibility. For the staggered fermion formulation most commonly used in the lattice calculations at non-zero temperature these quantities can be written as follows:

$$
\begin{aligned}
& \langle\bar{\psi} \psi\rangle_{q, x}=\frac{1}{4} \frac{1}{N_{\sigma}^{3} N_{\tau}} \operatorname{Tr}\left\langle D_{q}^{-1}\right\rangle, \\
& \chi_{m, q}(T)=n_{f} \frac{\partial\langle\bar{\psi} \psi\rangle_{q, \tau}}{\partial m_{l}}=\chi_{q, d i s c}+\chi_{q, c o n} q=l, s,
\end{aligned}
$$

where the subscript $x=\tau$ and $x=0$ will denote the expectation value at finite and zero temperature, respectively. Furthermore, $D_{q}=m_{q} \cdot 1+D$ is the fermion matrix in the canonical normalization and $n_{f}=2$ and 1 for light and strange quark. In Eq. (5.2) we made explicit that chiral susceptibility is the sum of connected and disconnected Feynman diagrams. The disconnected and connected contributions can be written as

$$
\begin{aligned}
& \chi_{q, \text { disc }}=\frac{n_{f}^{2}}{16 N_{\sigma}^{3} N_{\tau}}\left\{\left\langle\left(\operatorname{Tr} D_{q}^{-1}\right)^{2}\right\rangle-\left\langle\operatorname{Tr} D_{q}^{-1}\right\rangle^{2}\right\}, \\
& \chi_{q, \text { con }}=-\frac{n_{f}}{4} \operatorname{Tr} \sum_{x}\left\langle D_{q}^{-1}(x, 0) D_{q}^{-1}(0, x)\right\rangle, \quad q=l, s .
\end{aligned}
$$

The disconnected part of the light quark susceptibility describes the fluctuations in the light quark condensate and is directly analogous to the fluctuations in the order parameter of an $O(N)$ spin model. The second term $\left(\chi_{q, c o n}\right)$ arises from the explicit quark mass dependence of the chiral condensate and is the expectation value of the volume integral of the correlation function of the (isovector) scalar operator $\bar{\psi} \psi$. Let me note that in the massless limit only $\chi_{l, \text { disc }}$ diverges.

\subsection{The temperature dependence of the chiral condensate}

The chiral condensate needs a multiplicative, and also an additive renormalization if the quark mass is non-zero. Therefore the subtracted chiral condensate is considered

$$
\Delta_{l, s}(T)=\frac{\langle\bar{\psi} \psi\rangle_{l, \tau}-\frac{m_{l}}{m_{s}}\langle\bar{\psi} \psi\rangle_{s, \tau}}{\langle\bar{\psi} \psi\rangle_{l, 0}-\frac{m_{l}}{m_{s}}\langle\bar{\psi} \psi\rangle_{s, 0}} .
$$



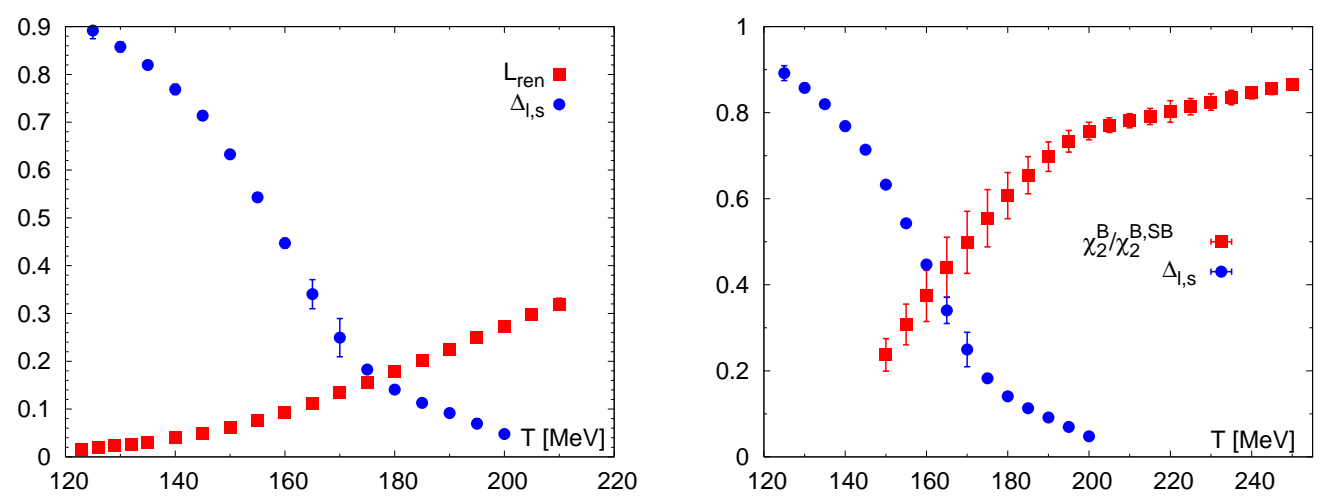

Figure 5: The subtracted chiral condensate calculated with $H I S Q$ action in the continuum limit compared to the renormalized Polyakov loop (left) and light quark number fluctuation (right). The continuum results for $\Delta_{l, s}$ and $L_{r e n}$ have been taken from Ref. [48], while the continuum results for $\chi_{2}^{B}$ have been taken from Ref. [39].

In Fig. 5 I show continuum results for $\Delta_{l, s}$ calculated with $H I S Q$ action and compared to the renormalized Polyakov loop and baryon number fluctuation previously discussed in relation to the deconfining transition. The rapid increase in $\chi_{2}^{B}$ happens roughly in the same temperature interval where $\Delta_{l, s}$ shows a rapid decrease, while it is difficult to make similar statements for $L_{r e n}$ due to its very smooth behavior. However, it is clear from Fig. 5 that $L_{r e n}$ is very far from unity for temperatures where $\Delta_{l, s}$ is very small.

Another way to get rid of the multiplicative and additive renormalization is to subtract the zero temperature condensate and multiply the difference by the strange quark mass, i.e. consider the following quantity

$$
\Delta_{q}^{R}=d+2 m_{s} r_{1}^{4}\left(\langle\bar{\psi} \psi\rangle_{q, \tau}-\langle\bar{\psi} \psi\rangle_{q, 0}\right), \quad q=l, s
$$

The factor $r_{1}^{4}$ was introduce to make the combination dimensionless. Here $r_{1}$ is the scale parameter defined from the zero temperature static potential [19]. It is convenient to choose the normalization constant to be the light quark condensate for $m_{l}=0$ multiplied by $m_{s} r_{1}^{4}$. In Fig. 6 the renormalized quark condensate is shown as function of the temperature for HISQ and stout actions. We see a crossover behavior for temperature of $(150-160) \mathrm{MeV}$, where $\Delta_{l}^{R}$ drops by $50 \%$. The difference between the stout and HISQ results is a quark mass effect. Calculations for HISQ action were performed for $m_{\pi}=160 \mathrm{MeV}$, while the stout calculations were done for the physical quark mass. For a direct comparison with stout results, we extrapolate the HISQ data in the light quark mass and also take care of the residual cutoff dependence in the HISQ data. This was done in Ref. [19] and the results are shown in the figure as black diamonds demonstrating a good agreement between $H I S Q$ and stout results. Contrary to $\Delta_{l}^{R}$ the renormalized strange quark condensate $\Delta_{s}^{R}$ shows only a gradual decrease over a wide temperature interval dropping by $50 \%$ only at significantly higher temperatures of about $190 \mathrm{MeV}$. The subtracted chiral condensate has also been calculated using Wilson and overlap formulations [20,24]. These calculations show good agreement with staggered results at the corresponding values of the pion mass. 

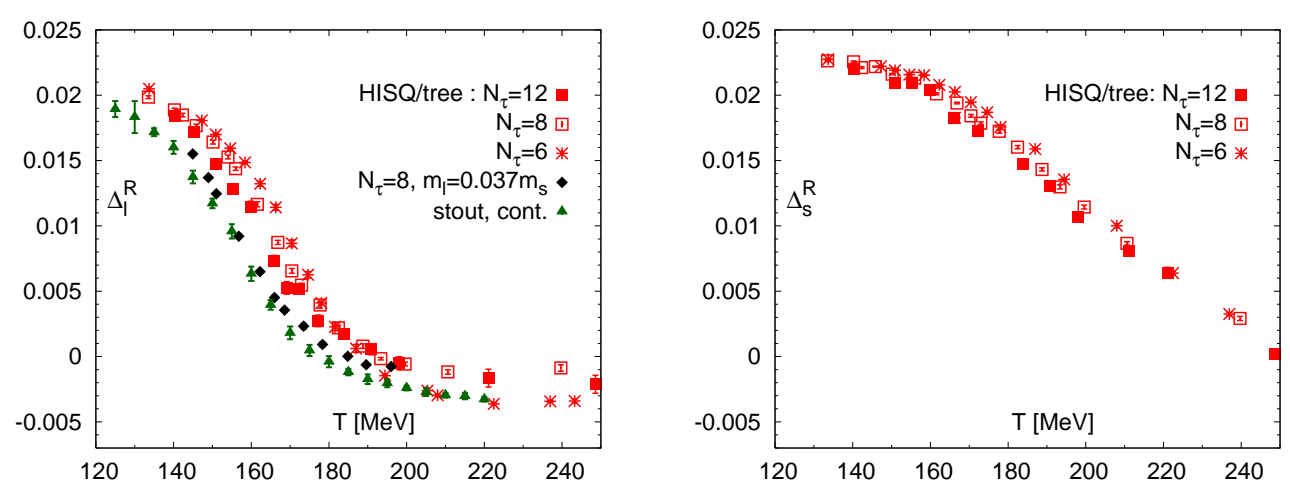

Figure 6: The renormalized chiral condensate $\Delta_{l}^{R}$ for the $H I S Q$ action with $m_{l} / m_{s}=0.05$ is compared to the stout data. In the right panel, we show the renormalized strange quark condensate $\Delta_{s}^{R}$ for the HISQ action.

\section{2 $\mathrm{O}(\mathrm{N})$ scaling and the transition temperature}

In the vicinity of the chiral phase transition, the free energy density may be expressed as a sum of a singular and a regular parts,

$$
f=-\frac{T}{V} \ln Z \equiv f_{\text {sing }}(t, h)+f_{\text {reg }}\left(T, m_{l}, m_{s}\right) .
$$

Here $t$ and $h$ are dimensionless couplings that control deviations from criticality. They are related to the temperature $T$ and the light quark mass $m_{l}$ as

$$
t=\frac{1}{t_{0}} \frac{T-T_{c}^{0}}{T_{c}^{0}} \quad, \quad h=\frac{1}{h_{0}} H \quad, \quad H=\frac{m_{l}}{m_{s}},
$$

where $T_{c}^{0}$ denotes the chiral phase transition temperature, i.e., the transition temperature at $H=0$. The scaling variables $t, h$ are normalized by two parameters $t_{0}$ and $h_{0}$, which are unique to QCD and similar to the low energy constants in the chiral Lagrangian. These need to be determined together with $T_{c}^{0}$. In the continuum limit, all three parameters are uniquely defined, but depend on the value of the strange quark mass.

The singular contribution to the free energy density is a homogeneous function of the two variables $t$ and $h$. Its invariance under scale transformations can be used to express it in terms of a single scaling variable

$$
z=t / h^{1 / \beta \delta}=\frac{1}{t_{0}} \frac{T-T_{c}^{0}}{T_{c}^{0}}\left(\frac{h_{0}}{H}\right)^{1 / \beta \delta}=\frac{1}{z_{0}} \frac{T-T_{c}^{0}}{T_{c}^{0}}\left(\frac{1}{H}\right)^{1 / \beta \delta}
$$

where $\beta$ and $\delta$ are the critical exponents of the $O(N)$ universality class and $z_{0}=t_{0} / h_{0}^{1 / \beta \delta}$. Thus, the dimensionless free energy density $\tilde{f} \equiv f / T^{4}$ can be written as

$$
\tilde{f}\left(T, m_{l}, m_{s}\right)=h^{1+1 / \delta} f_{f}(z)+f_{r e g}\left(T, H, m_{s}\right),
$$

where $f_{f}$ is the universal scaling function and the regular term $f_{\text {reg }}$ gives rise to scaling violations. This regular term can be expanded in a Taylor series around $(t, h)=(0,0)$. 
It should be noted that the reduced temperature $t$ may depend on other couplings in the QCD Lagrangian which do not explicitly break chiral symmetry. In particular, it depends on light and strange quark chemical potentials $\mu_{q}$, which in leading order enter only quadratically,

$$
t=\frac{1}{t_{0}}\left(\frac{T-T_{c}^{0}}{T_{c}^{0}}+\sum_{q=l, s} \kappa_{q}\left(\frac{\mu_{q}}{T}\right)^{2}+\kappa_{l s} \frac{\mu_{l}}{T} \frac{\mu_{s}}{T}\right) .
$$

The transition temperature can be defined as peaks in susceptibilities (response functions) that are second derivatives of the free energy density with respect to relevant parameters. Since there are two relevant parameters we can define three susceptibilities:

$$
\chi_{m, l}=\frac{\partial^{2} \tilde{f}}{\partial m_{l}^{2}}, \quad \chi_{t, l}=\frac{\partial^{2} \tilde{f}}{\partial t \partial m_{l}}, \quad \chi_{t, t}=\frac{\partial^{2} \tilde{f}}{\partial t^{2}} .
$$

Thus three different pseudo-critical temperatures $T_{m, l}, T_{t, l}$ and $T_{t, t}$ can be defined. In the vicinity of the critical point the behavior of these susceptibilities is controlled by three universal scaling function that can be derived from $f_{f}$. In the chiral limit $T_{m, l}=T_{t, l}=T_{t, t}=T_{c}^{0}$. There is, however, an additional complication for $O(N)$ universality class: while $\chi_{m, l}$ and $\chi_{t, l}$ diverge at the critical point for $m_{l} \rightarrow 0$

$$
\chi_{m, l} \sim m_{l}^{1 / \delta-1}, \quad \chi_{t, l} \sim m_{l}^{(\beta-1) / \beta \delta}
$$

$\chi_{t, t}$ is finite because $\alpha<0$ for $O(N)$ models $\left(\chi_{t, t} \sim|t|^{-\alpha}\right)$. Therefore, one has to consider the third derivative of $\tilde{f}$ with respect to $t$ :

$$
\chi_{t, t, t}=\frac{\partial^{3} \tilde{f}}{\partial t^{3}} .
$$

In the vicinity of the critical point the derivatives with respect to $t$ can be estimated by taking the derivatives with respect to $\mu_{l}^{2}$, i.e. the response functions $\chi_{t, l}$ and $\chi_{t, t, t}$ are identical to the second Taylor expansion coefficient of the quark condensate and the sixth order expansion coefficient to the pressure, respectively. The former controls the curvature of the tran sition temperature as function of the quark chemical potential $\mu_{q}$ and was studied for $p 4$ action using $N_{\tau}=4$ and 8 lattices [63]. The later corresponds to the sixth order quark number fluctuation which is relate d to the deconfinement aspects of the transition. The fact that this quantity is sensitive to the chiral dynamics points to a relation between deconfining and chiral aspects of the transition. In the following I discuss the determination of the transition temperature defined as peak position of $\chi_{m, l}$, i.e. $T_{c}=T_{m, l}$.

\subsection{Determination of the transition temperature}

The $O(N)$ scaling described in the above subsection can be used to determine the pseudocritical temperature of the chiral transition. For the study of the $O(N)$ scaling it is convenient to consider the dimensionless order parameter

$$
M_{b}=m_{s} \frac{\langle\bar{\psi} \psi\rangle_{l}}{T^{4}}
$$

The subscript "b" refers to the fact that this is a bare quantity since the additive UV divergence is not removed. From the point of view of the scaling analysis this divergent term is just a regular 
contribution. For sufficiently small quark mass and in the vicinity of the transition region we can write

$$
M_{b}(T, H)=h^{1 / \delta} f_{G}\left(t / h^{1 / \beta \delta}\right)+f_{M, r e g}(T, H) .
$$

Here $f_{G}(z)$ is the scaling function related to $f_{f}$ and was calculated for $O(2)$ and $O(4)$ spin models $[64,65,66]$. The regular contribution can be parametrized as [19]

$$
\begin{aligned}
f_{M, r e g}(T, H) & =a_{t}(T) H \\
& =\left(a_{0}+a_{1} \frac{T-T_{c}^{0}}{T_{c}^{0}}+a_{2}\left(\frac{T-T_{c}^{0}}{T_{c}^{0}}\right)^{2}\right) H .
\end{aligned}
$$

Then we have the following behavior for the light chiral susceptibility

$$
\begin{aligned}
\frac{\chi_{m, l}}{T^{2}}= & \frac{T^{2}}{m_{s}^{2}}\left(\frac{1}{h_{0}} h^{1 / \delta-1} f_{\chi}(z)+\frac{\partial f_{M, r e g}(T, H)}{\partial H}\right), \\
& \text { with } f_{\chi}(z)=\frac{1}{\delta}\left[f_{G}(z)-\frac{z}{\beta} f_{G}^{\prime}(z)\right] .
\end{aligned}
$$

One then performs a simultaneous fit to the lattice data for $M_{b}$ and $\chi_{m, l}$ treating $T_{c}^{0}, t_{0}, h_{0}, a_{0}, a_{1}$ and $a_{2}$ as fit parameters [19]. This gives a good description of the quark mass and temperature dependence of $\chi_{m, l}$ and allows to determine accurately the peak position in $\chi_{m, l}$. Using this scaling analysis $T_{c}$ has been determined for asqtad and HISQ actions for different $N_{\tau}$. Having determined $T_{c}$ for $H I S Q$ and asqtad action for each $N_{\tau}$ a combined continuum extrapolation was performed using different assumption about the $N_{\tau}$ dependence of $T_{c}$ which resulted in the value [19]:

$$
T_{c}=(159 \pm 9) \mathrm{MeV}
$$

The analysis also demonstrated that HISQ and asqtad action give consistent results in the continuum limit. The Budapest-Wuppertal collaboration found $T_{c}=147(2)(3) \mathrm{MeV}, 157(3)(3) \mathrm{MeV}$ and $155(3)(3) \mathrm{MeV}$ defined as peak position in the chiral susceptibility, inflection points in $\Delta_{l, s}$ and $\Delta_{l}^{R}$ respectively [17]. These agree with the above value within errors. The peak position in $\chi_{\text {disc }}$ calculated using Domain Wall Fermions is also consistent with the $T_{c}$ value in Eq. (5.19).

\section{Conclusions}

In recent years significant progress has been made in lattice QCD calculations at non-zero temperature. Chiral and deconfining aspects of the QCD transition have been studied using improved staggered quark formulation allowing to control discretization effects. Some quantities have been calculated at small baryon density using Taylor expansion in chemical potentials. At sufficiently low temperatures lattice results can be understood in terms of hadron resonance gas model, while at high temperatures resummed perturbative calculations describe the lattice data quite well. For several quantities it has been shown that in the continuum limit different discretization schemes, including discretizations other than staggered, give consistent results. In particular, agreement has been reached on the value of the chiral transition temperature. There is still disagreement in the lattice calculation of the equation of state. 


\section{References}

[1] E. V. Shuryak, Phys. Lett. B 78 (1978) 150; D. Gross, R. Pisarski, L. Yaffe, Rev. Mod. Phys. 53, 43 (1981);

[2] B. Müller and J. Nagle, Ann. Rev. Nucl. Part. Sci. 56, 93 (2006)

[3] U. Wiedemann, Nucl. Phys. A, 830, 74c (2009)

[4] P. Petreczky, J. Phys. G 39 (2012) 093002

[5] O. Philipsen, arXiv:1207.5999 [hep-lat].

[6] C. W. Bernard et al., [MILC Collaboration], Phys. Rev. D 55 (1997) 6861

[7] A. Ali Khan et al., [CP-PACS Collaboration], Phys. Rev. D 64 (2001) 074510

[8] F. Karsch, E. Laermann and A. Peikert, Phys. Lett. B 478 (2000) 447;

[9] Y. Aoki et al. JHEP 0601, 089 (2006)

[10] M. Cheng et al., Phys. Rev. D 74, 054507 (2006)

[11] Y. Aoki et al., Phys. Lett. B 643 (2006) 46

[12] C. Bernard et al., Phys. Rev. D 75 (2007) 094505

[13] M. Cheng et al., Phys. Rev. D 77 (2008) 014511

[14] M. Cheng et al., Phys. Rev. D 81, 054504 (2010)

[15] A. Bazavov et al., Phys. Rev. D 80 (2009), 014504

[16] Y. Aoki, et al, JHEP 0906 (2009) 088

[17] S. Borsanyi et al. [Wuppertal-Budapest Collaboration], JHEP 1009, 073 (2010)

[18] S. Borsanyi et al, JHEP 1011, 077 (2010)

[19] A. Bazavov et al., Phys. Rev. D 85 (2012) 054503

[20] S. Borsanyi et al., JHEP 1208 (2012) 126

[21] T. Umeda et al. [WHOT-QCD Collaboration], PoS LATTICE 2012 (2012) 074

[22] B. B. Brandt, A. Francis, H. B. Meyer, H. Wittig and O. Philipsen, PoS LATTICE 2012 (2012) 073

[23] A. Bazavov et al. [HotQCD Collaboration], Phys. Rev. D 86 (2012) 094503

[24] S. Borsanyi et al., Phys. Lett. B 713 (2012) 342

[25] K. Orginos, D. Toussaint and R. L. Sugar [MILC Collaboration], Phys. Rev. D 60 (1999) 054503

[26] A. Bazavov et al. [for the HotQCD Collaboration], PoS LATTICE 2010, 169 (2010); W. Soldner [HotQCD Collaboration], PoS LATTICE 2010, 215 (2010)

[27] P. Petreczky [for HotQCD Collaboration], PoS LATTICE 2012 (2012) 069

[28] P. Huovinen and P. Petreczky, Nucl. Phys. A 837, 26 (2010)

[29] J. P. Blaizot, E. Iancu and A. Rebhan, Phys. Rev. Lett. 83 (1999) 2906;

[30] J. P. Blaizot, E. Iancu and A. Rebhan, Phys. Rev. D 63 (2001) 065003

[31] S.S. Gubser, I.R. Klebanov and I.I Tseytlin, Nucl. Phys. B 534 (1998) 202 
[32] J. O. Andersen, L. E. Leganger, M. Strickland and N. Su, arXiv:1009.4644 [hep-ph].

[33] S. .Borsanyi et. al, JHEP 1208 (2012) 053

[34] S. Ejiri, F. Karsch, E. Laermann and C. Schmidt, Phys. Rev. D 73 (2006) 054506

[35] C. Bernard et al, Phys. Rev. D 77 (2008) 014503

[36] A. Bazavov et al., Phys. Rev. Lett. 109 (2012) 192302

[37] M. Cheng et al., Phys. Rev. D 79 (2009) 074505

[38] S. Borsanyi et al. [Wuppertal-Budapest Collaboration], J. Phys. G G 38, 124060 (2011)

[39] A. Bazavov et al. [HotQCD Collaboration], Phys. Rev. D 86 (2012) 034509

[40] C. Schmidt, arXiv:1212.4283 [hep-lat]; arXiv:1212.4278 [hep-lat].

[41] P. Petreczky, P. Hegde and A. Velytsky [RBC-Bielefeld Collaboration], PoS LAT2009, 159 (2009)

[42] J. O. Andersen, S. Mogliacci, N. Su and A. Vuorinen, arXiv:1210.0912 [hep-ph].

[43] N. Haque, M. G. Mustafa and M. Strickland, arXiv:1212.1797 [hep-ph].

[44] P. Petreczky, C. Miao and A. Mocsy, Nucl. Phys. A 855, 125 (2011)

[45] see e.g. Y. Hatta and K. Fukushima, arXiv:hep-ph/0311267, and references therein.

[46] O. Kaczmarek et al., Phys. Lett. B 543, 41 (2002)

[47] S. Digal, S. Fortunato and P. Petreczky, Phys. Rev. D 68, 034008 (2003)

[48] A. Bazavov and P. Petreczky, arXiv:1301.3943 [hep-lat].

[49] E. Megias, E. Ruiz Arriola and L. L. Salcedo, Phys. Rev. Lett. 109 (2012) 151601

[50] P. Petreczky, Eur. Phys. J. C 43, 51 (2005)

[51] A. Bazavov, P. Petreczky and A. Velytsky, arXiv:0904.1748 [hep-ph].

[52] A. Bazavov, P. Petreczky and A. Velytsky, Phys. Rev. D 78, 114026 (2008)

[53] N. Brambilla et al., Phys. Rev. D 82, 074019 (2010)

[54] A. Bazavov and P. Petreczky, arXiv:1211.5638 [hep-lat].

[55] M. Cheng et al., Phys. Rev. D 78, 034506 (2008)

[56] F. Karsch, M. Oevers and P. Petreczky, Phys. Lett. B 442, 291 (1998)

[57] A. Cucchieri, F. Karsch and P. Petreczky, Phys. Lett. B 497, 80 (2001)

[58] A. Cucchieri, F. Karsch and P. Petreczky, Phys. Rev. D 64, 036001 (2001)

[59] S. Ejiri et al., Phys. Rev. D 80, 094505 (2009)

[60] Y. Aoki, et al., Nature 443, 675 (2006)

[61] R.D. Pisarski and F. Wilczek, Phys. Rev. D 49, 338 (1984)

[62] M. Cheng et al., Eur. Phys. J. C 71, 1564 (2011)

[63] O. Kaczmarek et al., Phys. Rev. D 83, 014504 (2011)

[64] J. Engels, S. Holtmann, T. Mendes and T. Schulze, Phys. Lett. B 492, 219 (2000).

[65] D. Toussaint, Phys. Rev. D 55, 362 (1997).

[66] J. Engels and T. Mendes, Nucl. Phys. B 572, 289 (2000).

[67] J. Engels, S. Holtmann, T. Mendes and T. Schulze, Phys. Lett. B 514, 299 (2001). 